\title{
TUMOUR FLUORESCENCE AND TUMOUR-ASSOCIATED FLUORESCENCE OF CHOROIDAL MELANOMAS
}

\author{
B. E. DAMATO \\ Liverpool
}

\begin{abstract}
SUMMARY
'Tumour fluorescence' occurs within the tumour itself and is inversely related to the degree of pigmentation of the tumour, whereas 'tumour-associated fluorescence' occurs within the retinal pigment epithelium and retina as a result of degenerative changes in these structures; these secondary changes can collectively be termed 'tumour-associated retinal pigment epitheliopathy'. Tumour fluorescence should be differentiated from tumour-associated fluorescence so as not to confuse naevus with melanoma and to facilitate the interpretation of angiographic changes after radiotherapy or photocoagulation of choroidal melanomas.
\end{abstract}

Fluorescein angiography is widely used as an aid to the diagnosis of choroidal melanomas and for assessing the response of such tumours to photocoagulation or radiotherapy. Nevertheless, the angiographic signs may be misleading if they are interpreted without due regard to the underlying pathology. The aim of this article is to emphasise the importance of distinguishing between fluorescence occurring within the tumour itself (i.e. tumour fluorescence) and fluorescence occurring as a result of degenerative changes in the tissues adjacent to the tumour (i.e. tumour-associated fluorescence).

\section{TUMOUR FLUORESCENCE}

Choroidal melanomas are known to have large sinusoidal blood vessels, which leak fluorescein into the tumour tissue and into the subretinal space through defects in the retinal pigment epithelium. ${ }^{1}$ If the tumour is amelanotic and directly visible through a large defect in the retinal pigment epithelium, then 'tumour fluorescence' readily occurs (Fig. 1), and the tumour vasculature may also be visible. $^{2,3}$ If, however, the tumour is deeply pigmented, then such fluorescence may be totally masked by the melanin within the tumour as shown in Figs. 2 and 3. 4.5

Correspondence to: Dr. Bertil E. Damato, PhD, FRCS, FCOphth, St. Paul's Eye Unit, Royal Liverpool University Hospital, Prescot Street, Liverpool L7 8XP, UK.
Tumour fluorescence is therefore inversely related to the degree of tumour pigmentation. Non-fluorescence of a choroidal melanoma is not necessarily due to photographic artefact ${ }^{6}$ or an overlying haemorrhage.

\section{TUMOUR-ASSOCIATED FLUORESCENCE}

Choroidal tumours tend to cause a variety of secondary degenerative changes in the adjacent and overlying retinal pigment epithelium and retina, which may collectively be termed 'tumour-associated retinal pigment epitheliopathy (TARPE) '. ${ }^{8}$ These changes, which are not specific for melanoma, include atrophy, proliferation and detachment of the retinal pigment epithelium, the accumulation of drusen and lipofuscin deposits, and also retinal detachment, atrophy and cyst formation (Figs. 1, 2 and 4). ${ }^{9}$ Several of these lesions occur in age-related macular degeneration and are well known to be hyperfluorescent. The fluorescence arising from such changes can be termed 'tumourassociated fluorescence' to be distinguished from tumour fluorescence. Although both types of fluorescence can occur simultaneously with amelanotic tumours, it is also possible for tumour-associated fluorescence to occur in isolation if the underlying tumour is deeply pigmented and non-fluorescent, as shown in Fig. 2. The fluorescein giving rise to tumour-associated fluorescence may arise from remnants of the choriocapillaris and not necessarily from the tumour circulation. For example, Fig. 5 shows an amelanotic choroidal melanoma with a defect in the overlying retinal pigment epithelium; the tumour-associated fluorescence commences before the tumour fluorescence.

It is not unusual for melanomas to have tapering edges that are covered by retinal pigment epithelium which is still normal and therefore semi-transparent. In such situations the angiographic appearances depend on the degree of tumour pigmentation. If the tumour is pigmented, then it will be visible as a hypofluorescent area silhouetted against the fluorescent choroid (Fig. 4). If, however, the tumour is non-pigmented, then it will be invisible because the fluorescence is similar to that of the adjacent choroid. 


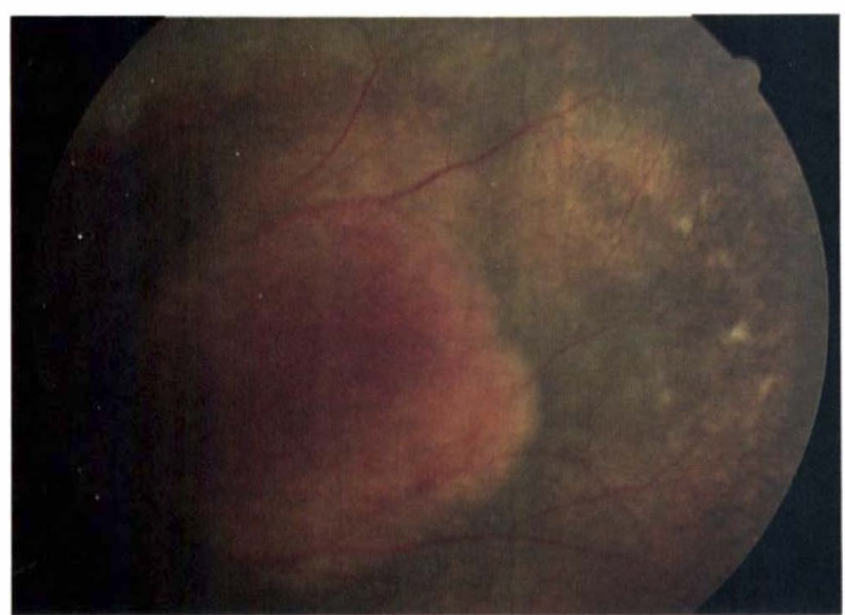

Fig. 1a

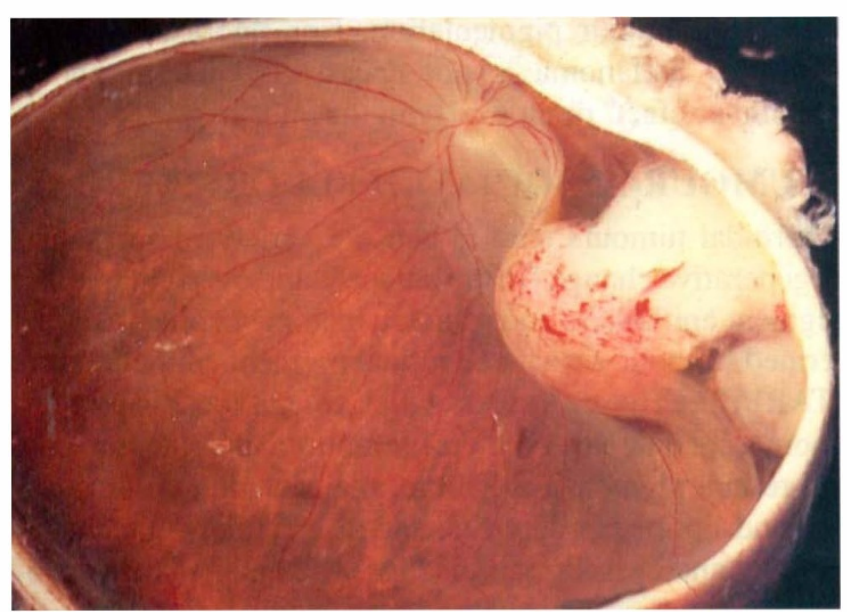

Fig. 1b

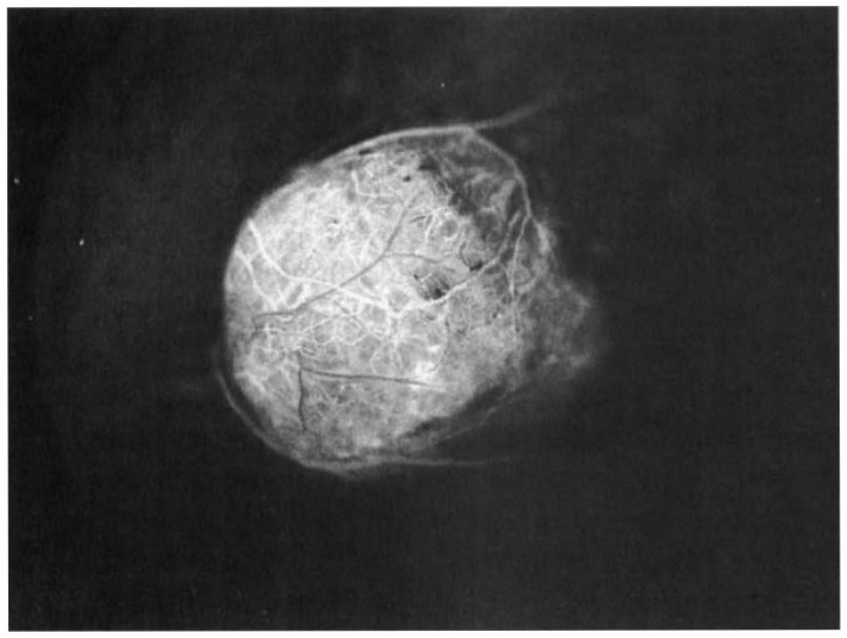

Fig. 1c

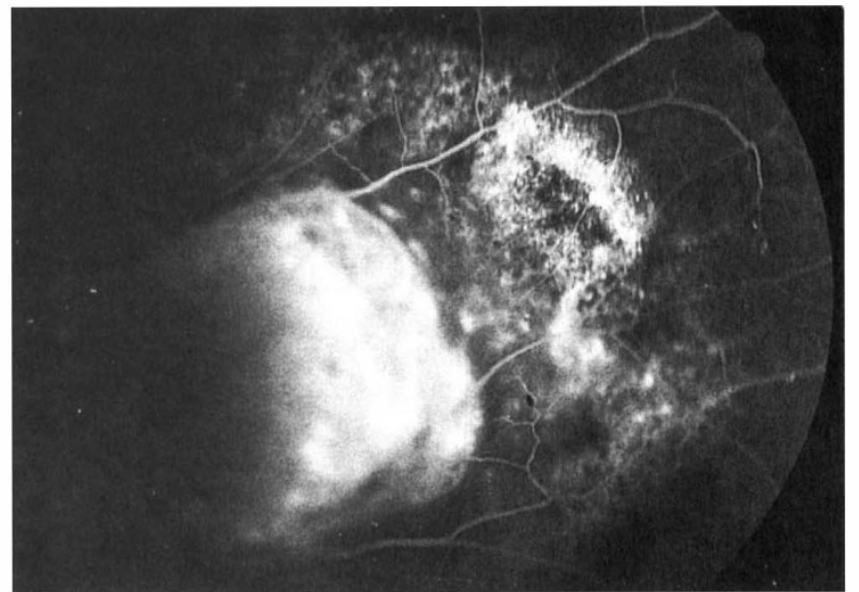

Fig. 1d

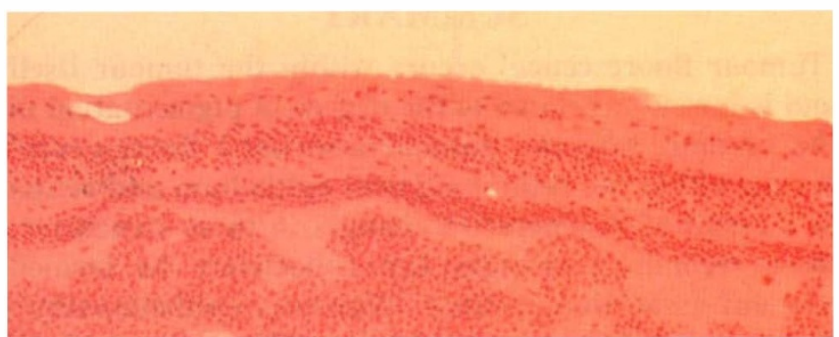

Fig. 1e

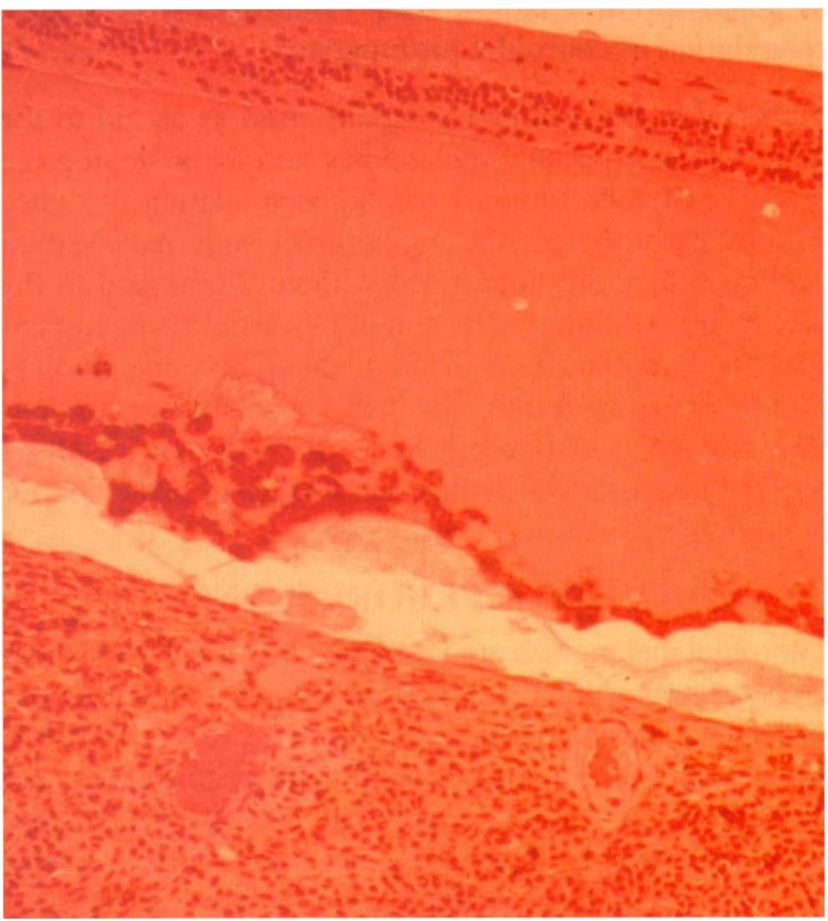

Fig. 1f

Fig. 1. Amelanotic choroidal melanoma, with trans-retinal pigment epithelial extension. (a) Fundus photograph showing exposed tumour, which is amelanotic, and base of tumour, which is covered by proliferated and hyperpigmented retinal pigment epithelium. (b) Macroscopic photograph of specimen showing the amelanotic nature of the tumour. (c) Hyperfluorescent retinal and choroidal vessels (i.e. 'dual circulation'). (d) Hyperfluorescent blebs at base of tumour. (e) Light micrograph showing apical portion of tumour, which has broken through the retinal pigment epithelium into the subretinal space. $(f)$ Multiple retinal pigment epithelial detachments and drusen at the level of the retinal pigment epithelium at base of tumour, which account for the hyperfluorescent spots. 


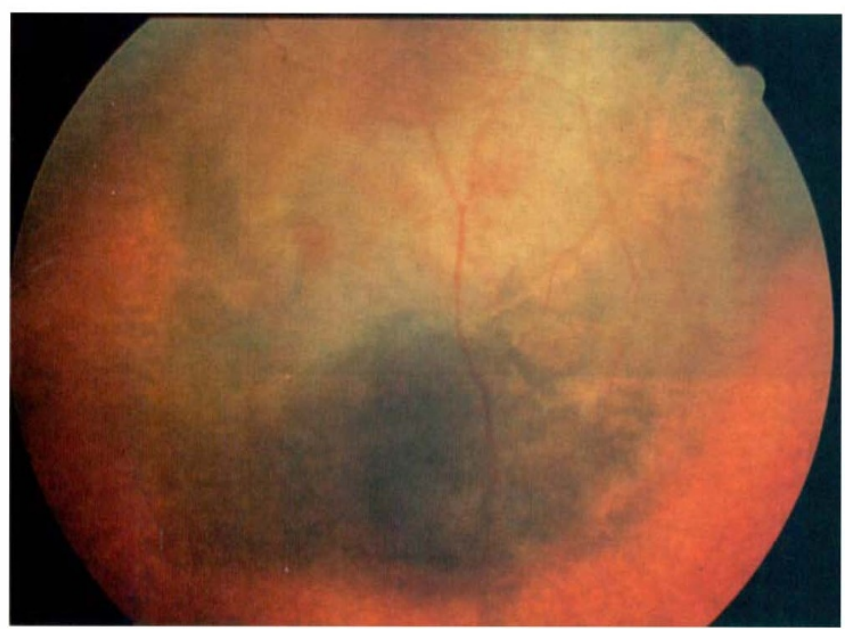

Fig. 2a

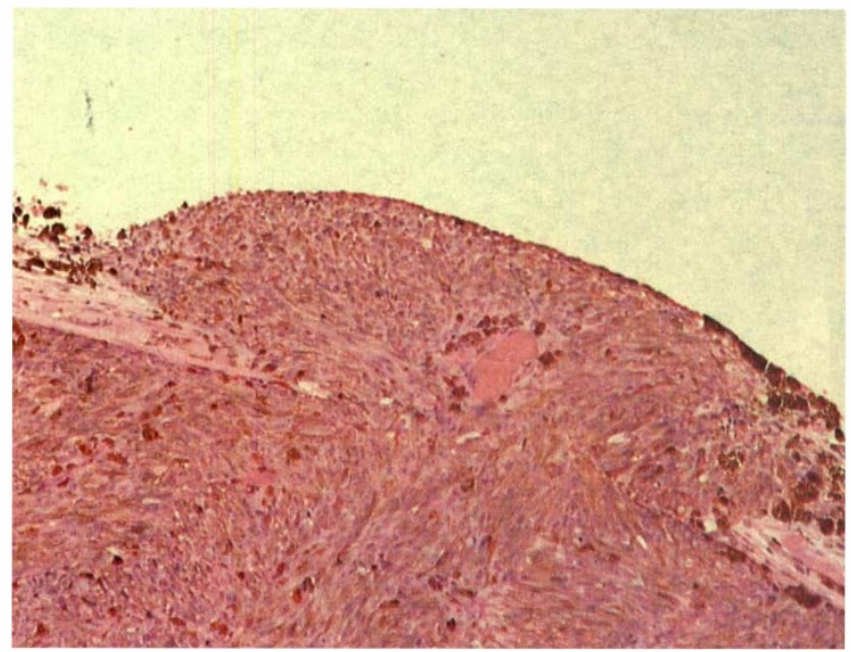

Fig. 2c

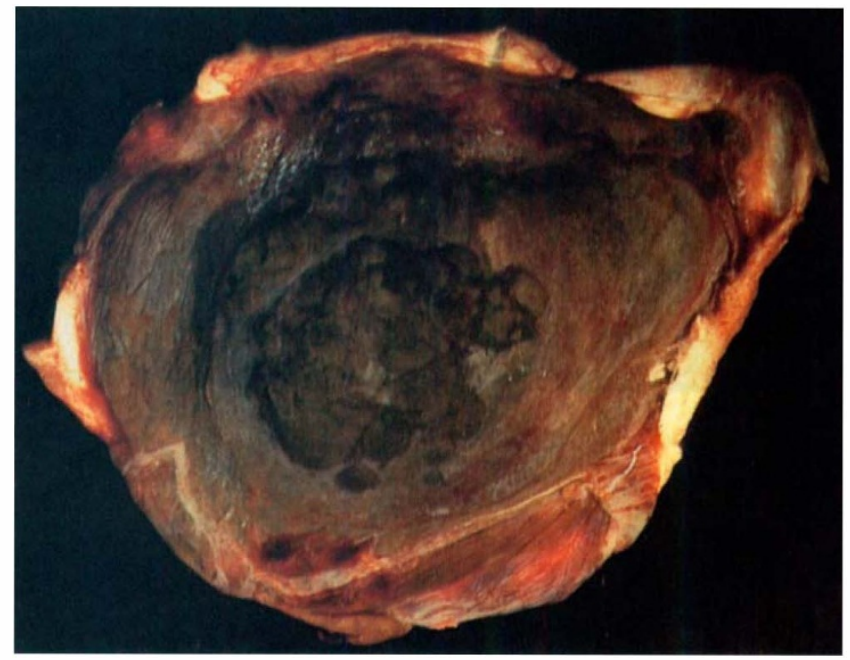

Fig. 2b

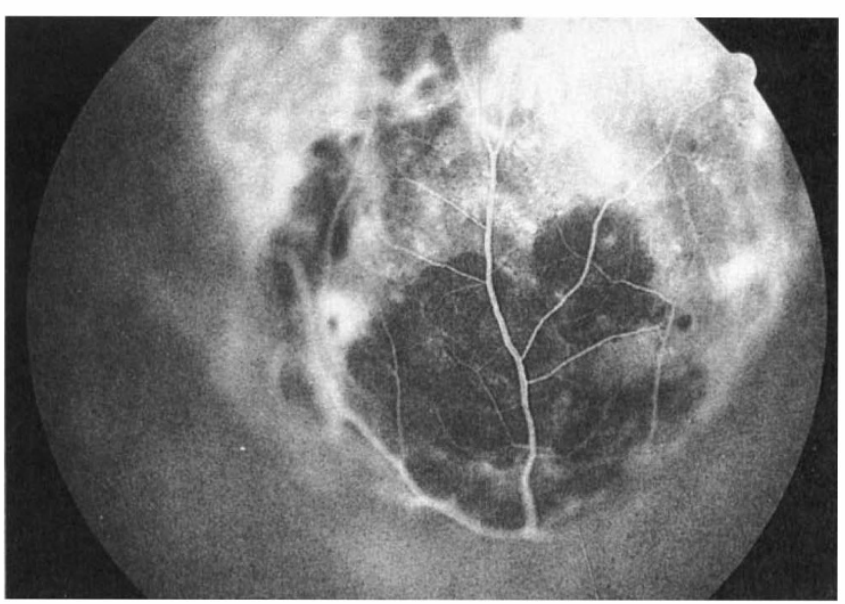

Fig. 2d

Fig. 2. Pigmented choroidal melanoma with rupture of retinal pigment epithelium and Bruch's membrane. (a) Colour photograph. (b) Locally excised specimen showing darker areas which have ruptured retinal pigment epithelium. (c) Light micrograph showing lateral spread of the tumour anterior to Bruch's membrane and retinal pigment epithelium. (d) Fluorescein angiograph showing hypofluorescent areas corresponding to exposed parts of the tumour, which are masking retinal pigment epithelial hyperfluorescence. 


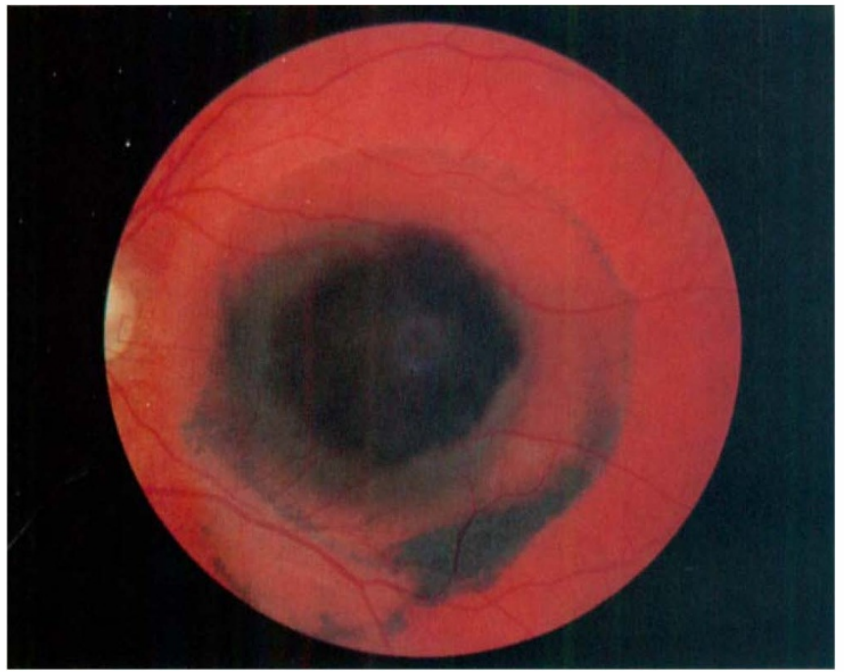

Fig. 3a
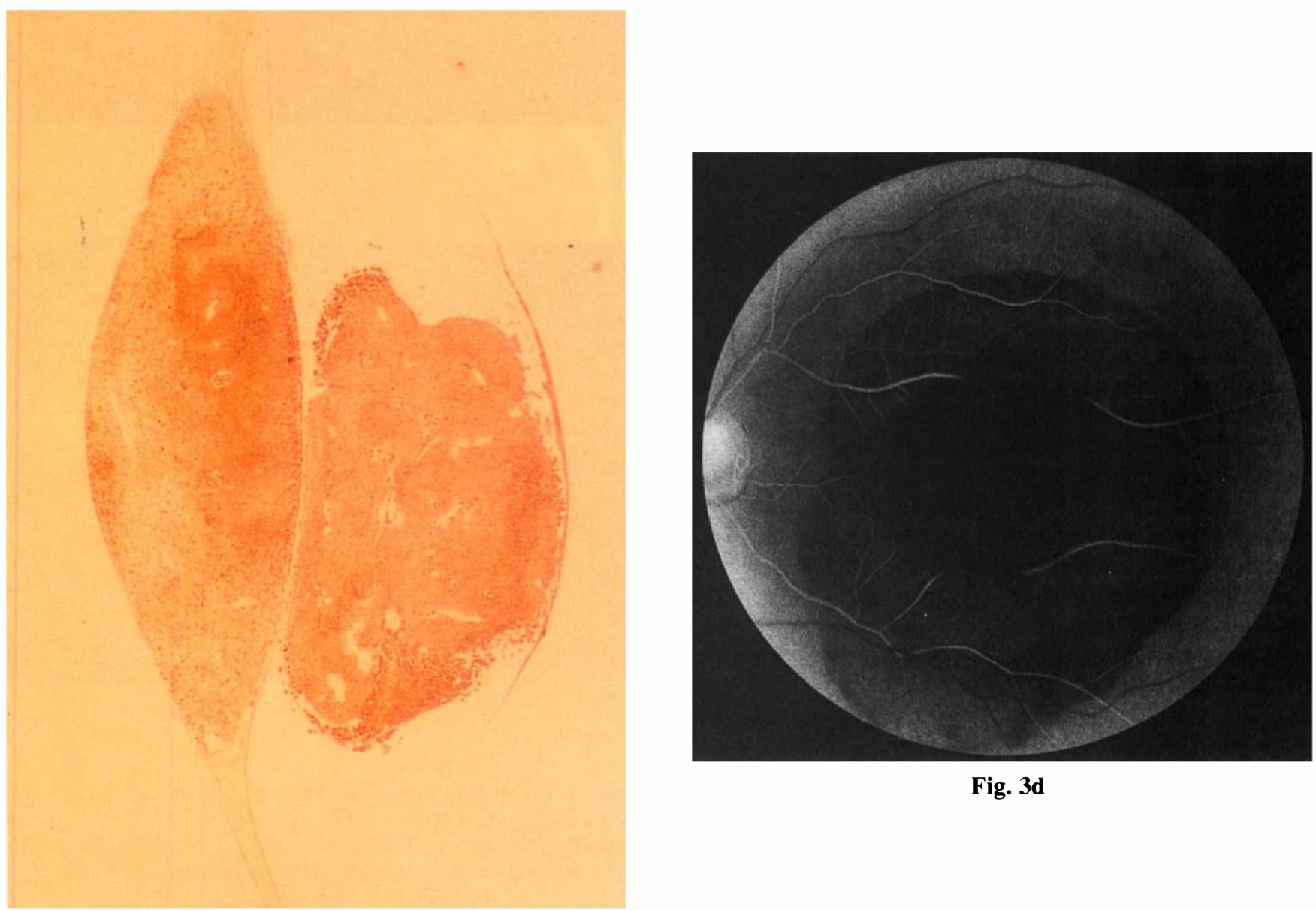

Fig. 3d

Fig. 3c

Fig. 3. Deeply pigmented collar-stud choroidal melanoma. (a) Fundus photograph showing intra-retinal invasion and subretinal seeding of tumour cells. (b) Macroscopic photograph of excised eye showing deeply pigmented nature of tumour. (c) Light micrograph, without any staining, showing melanin pigment in tumour and invaded retina. (d) Fluorescein angiograph showing total masking of tumour fluorescence by melanin. 


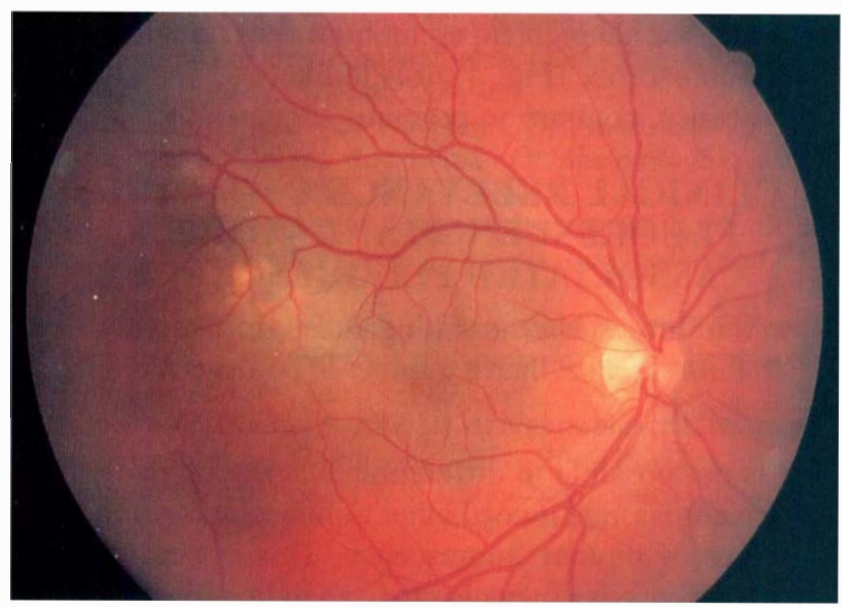

Fig. 4a

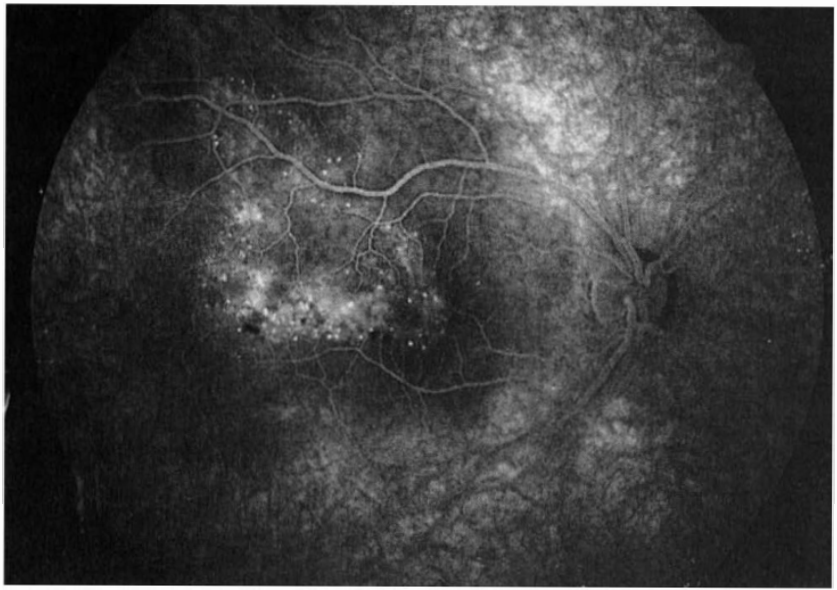

Fig. 4c

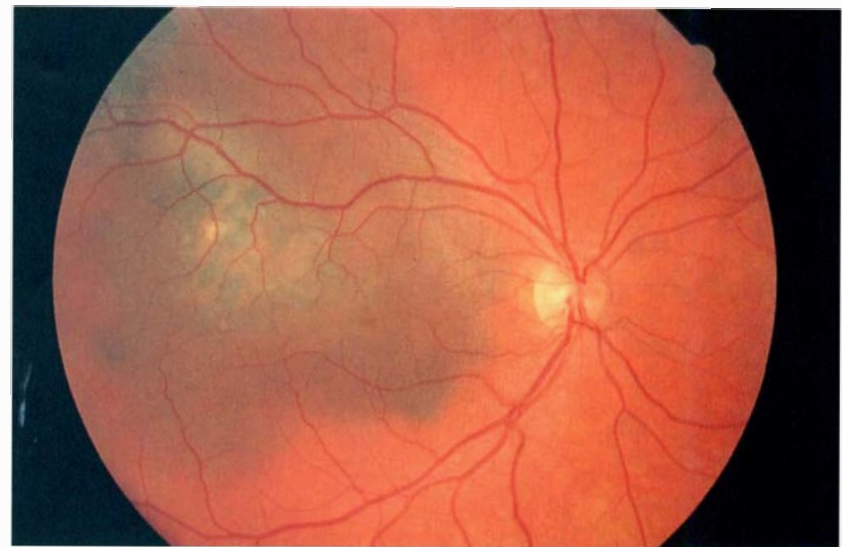

Fig. 4b

Fig. 4. A flat choroidal melanoma at the right macula. (a) Colour photograph on presentation. (b) Colour photograph 2 months later showing lateral extension of the tumour, which confirms the malignant nature of the entire lesion. (c) Fluorescein angiograph showing irregular hyperfluorescence and drusen in the central part of the tumour, surrounded by hypofluorescent tumour, which is silhouetted against the fluorescence of the normal choroid. (d) Irregularity of retinal pigment epithelium with the accumulation of drusen in the hyperfluorescent part of tumour. (e) Normal retinal pigment epithelium overlying the hypofluorescent part of tumour. The retina artefactually separated from the retinal pigment epithelium during histopathological processing.

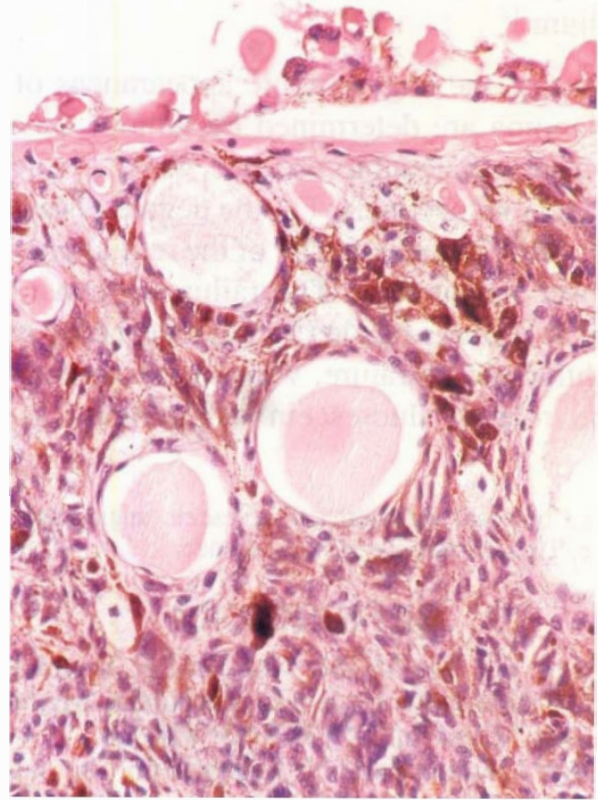

Fig. 4d

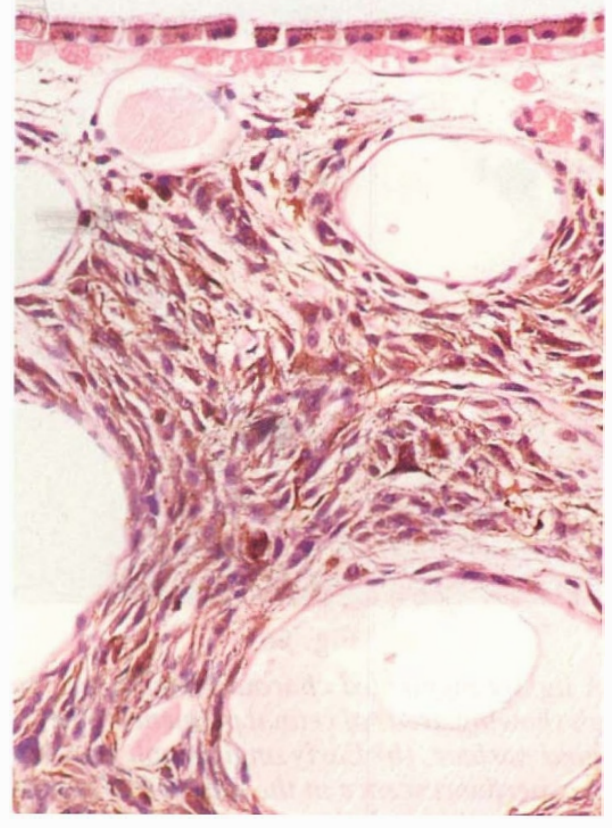

Fig. 4e 


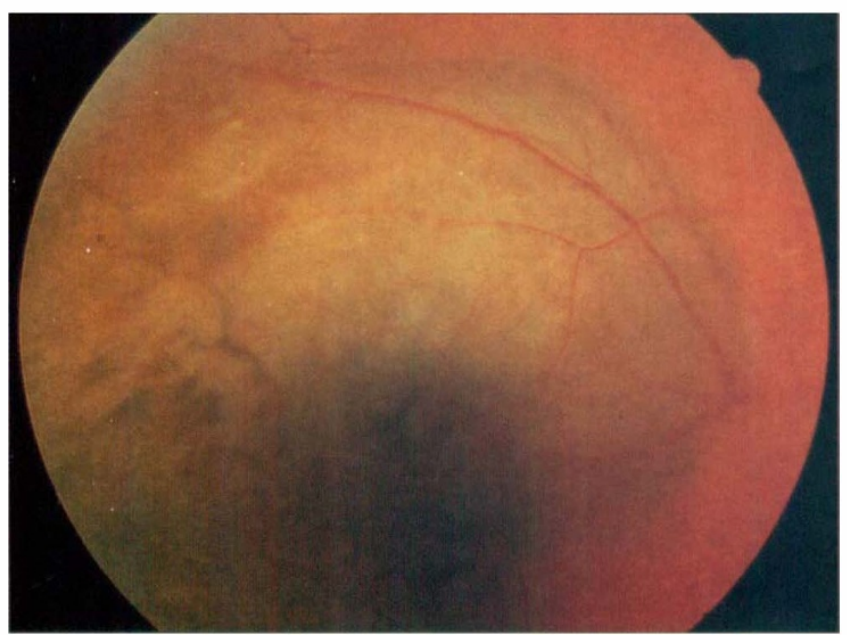

Fig. 5a

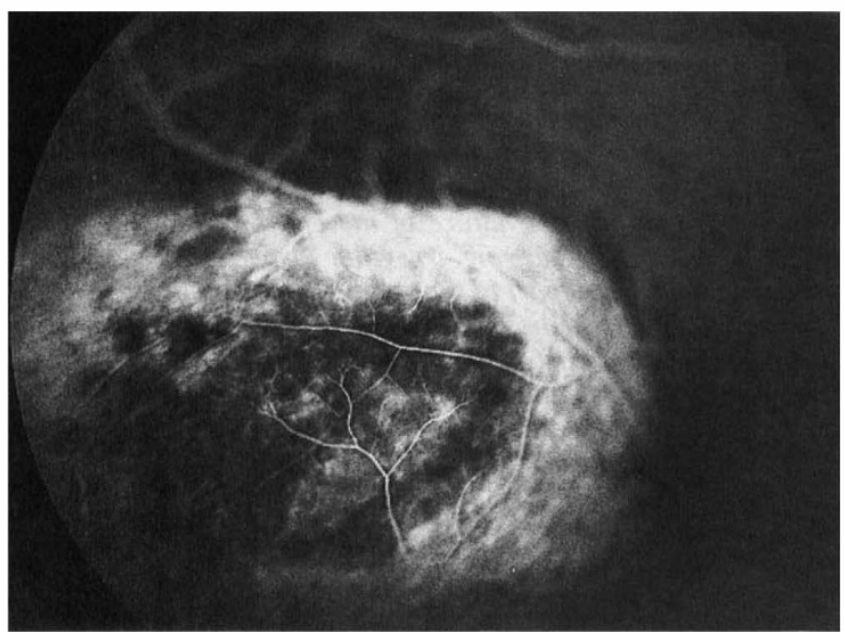

Fig. 5b

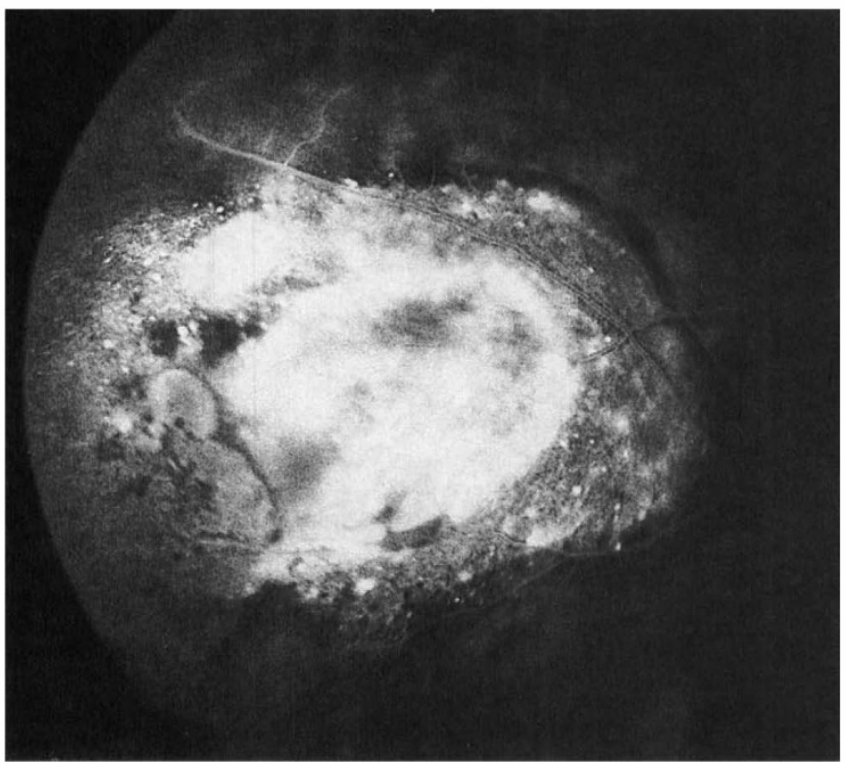

Fig. 5c

Fig. 5. A lightly pigmented choroidal melanoma. (a) Colour photograph showing areas of retinal pigment epithelial atrophy on the tumour surface. (b) Early angiograph showing tumourassociated hyperfluorescence in the absence of tumour fluorescence. (c) Late angiograph showing tumour fluorescence when tumour-associated fluorescence has started to fade.
Since the normal pigment epithelium does not block choroidal fluorescence (Fig. 4), it is unlikely to block tumour fluorescence, contrary to previous suggestions. ${ }^{10}$

\section{CLINICAL RELEVANCE OF TUMOUR FLUORESCENCE AND TUMOUR- ASSOCIATED FLUORESCENCE}

If tumour fluorescence is distinguished from tumour-associated fluorescence, then a number of points can readily be appreciated:

1. It is not possible to differentiate choroidal naevi from melanomas, and indeed other tumours, according to the degree of tumour fluorescence. This is because such fluorescence is not necessarily an indication of the vascularity, and hence the metabolic activity, of a tumour as previously suggested ${ }^{11}$ but more likely to be determined by the pigmentation of the tumour and the nonspecific degenerative changes in the overlying retinal pigment epithelium.

2. Hypofluorescence at the margin of a melanoma is unlikely to represent the original naevus, as previously suggested, ${ }^{11}$ but is more probably a flat extension of a pigmented melanoma and should be treated as such.

3. If a melanoma is amelanotic then it is not possible to define the extent of the tumour by angiography, because the tumour fluorescence and choroidal fluorescence are similar.

4. Hypofluorescence of a choroidal melanoma is most likely to be due to tumour pigmentation when the overlying retinal pigment epithelium is either absent or normal and therefore semi-transparent.

5. Although loss of fluorescence after photocoagulation has been attributed to tumour destruction, ${ }^{4}$ non-fluorescence may merely be due to obliteration of the tumour-associated fluorescence by destruction of the choriocapillaris, retina and retinal pigment epithelium. $^{12}$

In summary, the angiographic appearances of a choroidal melanoma are determined by tumour fluorescence and tumour-associated fluorescence, which in turn depend on two factors respectively: (1) the degree of tumour pigmentation, and (2) the integrity of the retinal pigment epithelium over the tumour. The failure to consider these pathological features has led to fallacies being published in the ophthalmic literature, which have tended to exaggerate the role of fluorescein angiography in clinical practice.

Key words: Choroidal melanoma, Fluorescein angiography, Tumour fluorescence, Tumour-associated fluorescence.

\section{REFERENCES}

1. McMahon RT, Tso MOM, McLean IW: Histologic localization of sodium fluorescein in choroidal malignant melanomas. Am J Ophthalmol 1977, 83: 836-46.

2. Charamis J, Katsourakis N, Mandras G: The study of the cerebroretinal circulation by intravenous fluorescein injection. Am J Ophthalmol 1966, 61: 1078-80. 
3. Augsburger JA, Golden MI, Shields JA: Fluorescein angiography of choroidal malignant melanomas with retinal invasion. Retina 1984, 4: 232-41.

4. Oosterhuis JA and van Waveren CW: Fluorescein angiography in malignant melanoma. Ophthalmologica 1968, 156: $101-16$.

5. Gass JDM: Fluorescein angiography: an aid to the differential diagnosis of intraocular tumors. Int Ophthalmol Clin 1972, 12: 85-120.

6. Edwards WC, Layden WE, Macdonald R, Jr: Fluorescein angiography of malignant melanoma of the choroid. Am J Ophthalmol 1969, 68: 797-808.

7. Nadel A, O'Connor P, Lincoff H: Nonfluorescent choroidal melanoma. Am J Ophthalmol 1970, 70: 748-52.

8. Damato BE and Foulds WS: Tumour-associated retinal pigment epitheliopathy. Eye 1990, 4: 382-7.
9. Wallow IHL and Ts'o MOM: Proliferation of the retinal pigment epithelium over malignant choroidal tumors: a light and electron microscopic study. Am J Ophthalmol 1972, 73: 914-26.

10. Shields JA, Annesley WH, Jr, Totino JA: Nonfluorescent malignant melanoma of the choroid diagnosed with the radioactive phosphorus uptake test. Am J Ophthalmol 1975, 79: $634-40$.

11. Hayreh SS: Choroidal melanomata: fluorescence angiographic and histopathological study. Br J Ophthalmol 1970, 54: $145-60$.

12. Damato BE: Why do choroidal melanomas fluoresce on angiography? In: Bornfeld B, Gragoudas ES, Hopping W, Lommatzsch PK, Wessing A, Zografos L, eds. Tumors of the eye. Amsterdam/New York: Kugler Publications, 1991: 223-30. 